EPJ Web of Conferences 52, 10001 (2013)

DOI: 10.1051/epjconf/20135210001

(C) Owned by the authors, published by EDP Sciences, 2013

\title{
The origin of cosmic rays and $\mathrm{TeV}$ gamma-ray astronomy
}

\author{
Gernot Maier $^{1, a}$ \\ ${ }^{1}$ DESY, Platanenallee 7, 15738 Zeuthen, Germany
}

\begin{abstract}
Cosmic rays are accelerated to high energies in Galactic and extragalactic objects like Supernova remnants (SNR) and active galactic nuclei (AGN). How these accelerators work and how efficient they accelerate different types of particles to energies of $10^{15} \mathrm{eV}$ or beyond, is 100 years after the discovery of cosmic rays by Victor Hess, still unknown. Gamma rays trace cosmic rays at their site of acceleration and give crucial information on the nature and inner workings of these extreme objects. Gamma rays can be used to find the sources of cosmic rays and to determine their type, age and dynamics. We review in these proceedings the observational techniques and recent findings on gamma-ray emission from Supernova remnants.
\end{abstract}

\section{Introduction}

In 1912, the Austrian physicist Victor F. Hess discovered by means of several balloon ascents the particle population in the universe of highest energy: cosmic rays. Their energy spectrum can be described by a power law covering 10 order of magnitudes in energy (up to $10^{20} \mathrm{eV}$ ) and about 30 orders of magnitude in flux. Cosmic rays consist mainly of nuclei like protons, helium and heavier types up to iron. Supernova remnants (SNRs) have been the prime candidates as sources of galactic cosmic rays since the 1960s. The working hypothesis is that a $10 \%$ conversion of the blast wave's energy into relativistic particles during the lifetime of a SNR are enough to fill the Galaxy with cosmic rays up to about $10^{15} \mathrm{eV}$. Today, there is much unequivocal evidence for the acceleration of cosmic rays in SNRs from observations at different wavelengths: the expansion measurements in X-rays [1]; observation of magnetic field amplification as byproduct of particle acceleration [2]; dynamical measurements of shock positions and shock temperature [3] and nonthermal emission in gamma rays (e.g.[4]). The mechanism of diffusive shock acceleration is a solid theoretical base for the production of cosmic rays by SNRs.

Gamma rays can be used to trace and identify highenergy particles at or close to their acceleration site. The different production mechanisms for gamma rays depend on energy and type of the parent particle population and the surrounding photon, magnetic and matter fields. Highenergy protons can produce gamma rays through protonproton or proton-gamma interaction, where secondary neutral pions decay into high-energy photons. Long-living secondary particles in these interactions can initiate particle cascades where photons can be produced through pair production or synchrotron radiation. High-energy leptons

\footnotetext{
ae-mail: gernot.maier@desy.de
}

loose energy mainly through synchrotron radiation (which is in general below a few $\mathrm{GeV}$ ), inverse Compton scattering on low-energy photons or Bremsstrahlung. We will show, using a few examples, in Chapter 3 what can be learned about the acceleration conditions in SNRs from observing them at gamma-ray energies.

$\mathrm{TeV}$ gamma-ray astronomy is a broad and dynamic field with a large number of source types observed (e.g. active galactic nuclei, binaries or pulsar wind nebula, diffuse emission, sources with no counterparts at other wavelengths). Significant progress has been made in the past decade in the understanding of our non-thermal Universe. We would like to refer the reader towards extensive reviews of the field, see e.g. [5-8].

\section{Observing gamma rays}

The energy spectrum of sources of high-energy gamma rays are approximately power-law like shape $d N / d E \propto$ $E^{-\Gamma}$ with spectral indices $\Gamma$ between 2 and 4 above a few tens of GeV. This results in very small photon fluxes, especially at high energies. As an example, the photon flux above $100 \mathrm{MeV}$ and $1 \mathrm{TeV}$ from the Crab Nebula, one of the strongest known gamma-ray emitter, is about 30 photons $/ \mathrm{m}^{2} /$ day and 10 photons $/ \mathrm{m}^{2} /$ year respectively. The Large Area Telescope (LAT) on the Fermi space telescope is currently the most sensitive detector for gamma rays in the $20 \mathrm{MeV}$ to $100 \mathrm{GeV}$ range. The pair conversion detector has an effective area of about 1 square meter, which is too small to detect the faint sources of gamma rays above hundreds of $\mathrm{GeV}$ [9]. Ground-based detection techniques which measure the extensive air showers from the interaction of the high-energy photons with Earth's atmosphere are most sensitive in this energy regime and are the main focus on in the following.

Imaging atmospheric Cherenkov telescopes (IACTs) collect the Cherenkov light emitted by relativistic parti- 


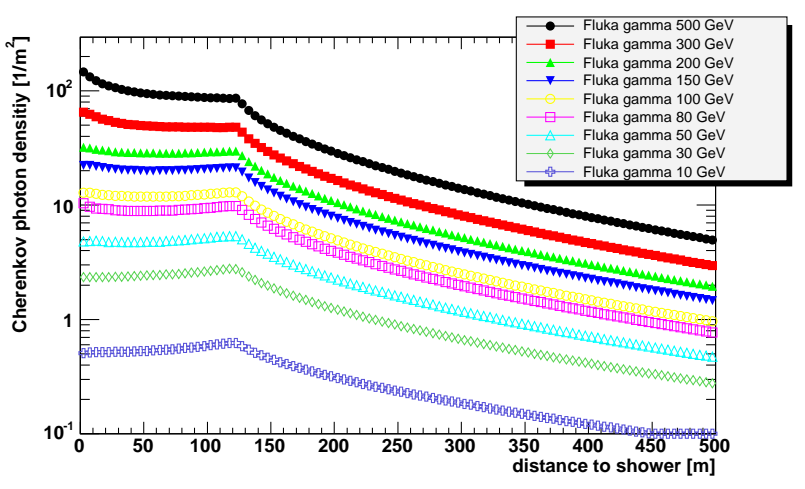

Figure 1. Average Cherenkov photon densities calculated by CORSIKA at an observation level of $1800 \mathrm{~m}$ a.s.l. for vertical showers with different primary energies.

cles (mainly electrons and positrons) in air showers. The Cherenkov photons are produced along the shower axis with an emission maximum at about $10 \mathrm{~km}$ above ground; they form a short nanoseconds long bluish $(300-550 \mathrm{~nm})$ light flash. The emission angle changes with altitude from about $0.1 \mathrm{deg}$ at $30 \mathrm{~km}$ height a.s.l. to about $1.3 \mathrm{deg}$ at sea level. This results in an approximately flat lateral distribution of Cherenkov photons on the ground, the so called light pool. The radius of the light pool is independent of the energy of the primary photon. It is at an observation level of $1800 \mathrm{~m}$ about $140 \mathrm{~m}$. The photon densities drop exponentially beyond this radius. Typical densities in the light pool are about $2-3$ photons $/ \mathrm{m}^{2}$ at $30 \mathrm{GeV}$ to thousands of photons $/ \mathrm{m}^{2}$ above a few TeVs, see Figure 1 . Placing a single telescope anywhere inside the light pool results in a large sensitive detection area of about $10^{5} \mathrm{~m}^{2}$.

To measure the faint and short flashes of Cherenkov light from air showers, IACTs consist of large mirrors (mirror area $>100 \mathrm{~m}^{2}$ ) to collect enough photons, pixelated cameras with $>500$ photomultiplier, sophisticated trigger systems and fast electronics. Each IACT measures a projection of the longitudinal development of the air shower in its camera. Using several telescopes and stereoscopic techniques allow the reconstruction of the direction of the incoming gamma ray; its energy can be estimated by the signal size. Fast trigger systems sensitive to the morphology of air showers are applied to suppress light from stars and other background sources. Readout rates for the current telescope systems are typically in the range of hundreds of $\mathrm{Hz}$, with most events being initiated by charged cosmic rays and not by gamma rays. Cosmic ray air showers are in general much more irregular with significantly more energy transferred to larger lateral distances from the shower axis. Most of these background events can therefore be eliminated by applying analysis cuts on the shape of their images in the camera.

The IACT technique was pioneered by the Whipple collaboration using a $10 \mathrm{~m}$ reflector located on Mt. Hopkins, Arizona [10]. The Whipple $10 \mathrm{~m}$ telescope was in operation from 1968-2011 and sensitive to gamma rays in the energy range from $200 \mathrm{GeV}$ to $20 \mathrm{TeV}$. The first very- high energy gamma ray sources where detected with this instrument: the Crab Nebula [10] and Mrk 421 [11]. Today, in 2012, three major telescope systems are in operation: H.E.S.S. ${ }^{1}$, MAGIC ${ }^{2}$ and VERITAS ${ }^{3}$.

H.E.S.S. phase $I$ is an array of four $12 \mathrm{~m}$ diameter IACTs operating since 2001 in Namibia. It is currently the only instrument on the southern hemisphere with an emphasis on observations of the inner regions of the Galaxy and the Galactic centre. A large fifth telescope with an optical dish diameter of $28 \mathrm{~m}$ has been added in Autumn 2012 to the array. This will lower the energy threshold of the system to below $50 \mathrm{GeV}$ and significantly enhance the sensitivity of H.E.S.S. to soft-spectrum transient events such as GRBs and flares from distant AGNs.

MAGIC is a two telescope system located on the Canary island La Palma. The MAGIC collaboration pioneered observations in the low-energy range between 25 $100 \mathrm{GeV}$, which are challenging for ground-based instruments due to the small amount of light emitted by these kind of air showers. The camera of MAGIC I was upgraded in 2012 to achieve a more uniform focal plane instrumentation (number of pixels increased from 576 to 1039); a new readout system and a new trigger system was also installed.

VERITAS is a four-telescope array of IACTs located in Southern Arizona close to the Whipple site at Mt. Hopkins. VERITAS has been in operation since 2007, the instrument was upgraded several times: 1. a single telescope was moved to achieve a more uniform array layout, resulting in an increase in sensitivity of $30 \%$; 2 . the replacement of the second level trigger by a new improved version for better suppression of the night sky background; 3. an upgrade of all four cameras with high-efficiency PMTs to achieve higher sensitivity and a significantly lower trigger threshold of about $70 \mathrm{GeV}$.

All observatories have similar characteristics: an energy range starting from $20-70 \mathrm{GeV}$ to $30 \mathrm{TeV}$, a sensitivity to detect a source with a flux of $1 \%$ of the Crab Nebula in about 20-40 hours, an effective area for gamma rays of $>10^{5} \mathrm{~m}^{2}$, an energy resolution of $15-20 \%$ and an angular resolution of $\approx 0.1 \mathrm{deg}$. The field of view of IACTs is $3-5$ deg wide, limiting their sensitivity to point-like or moderately extended sources. The duty cycle of ground-based Cherenkov instruments is restricted to dark nights resulting in about 1200 hours of observations per year. Observation during moderate moonlight conditions can extend the amount of observing time by about $30 \%$. These two limitations, the small field of view and duty cycle, means that large parts of the sky above $100 \mathrm{GeV}$ are not surveyed with high sensitivity.

Beside the hardware upgrades mentioned above, significant progress has been made in understanding signal and background measurements. Several new approaches for gamma-hadron separation and shower reconstruction result in typically $40 \%$ less observation time needed to

\footnotetext{
${ }^{1}$ http://www.mpi-hd.mpg.de/hfm/HESS/

${ }^{2}$ http://magic.mppmu.mpg.de/

${ }^{3}$ https://veritas.sao.arizona.edu/
} 


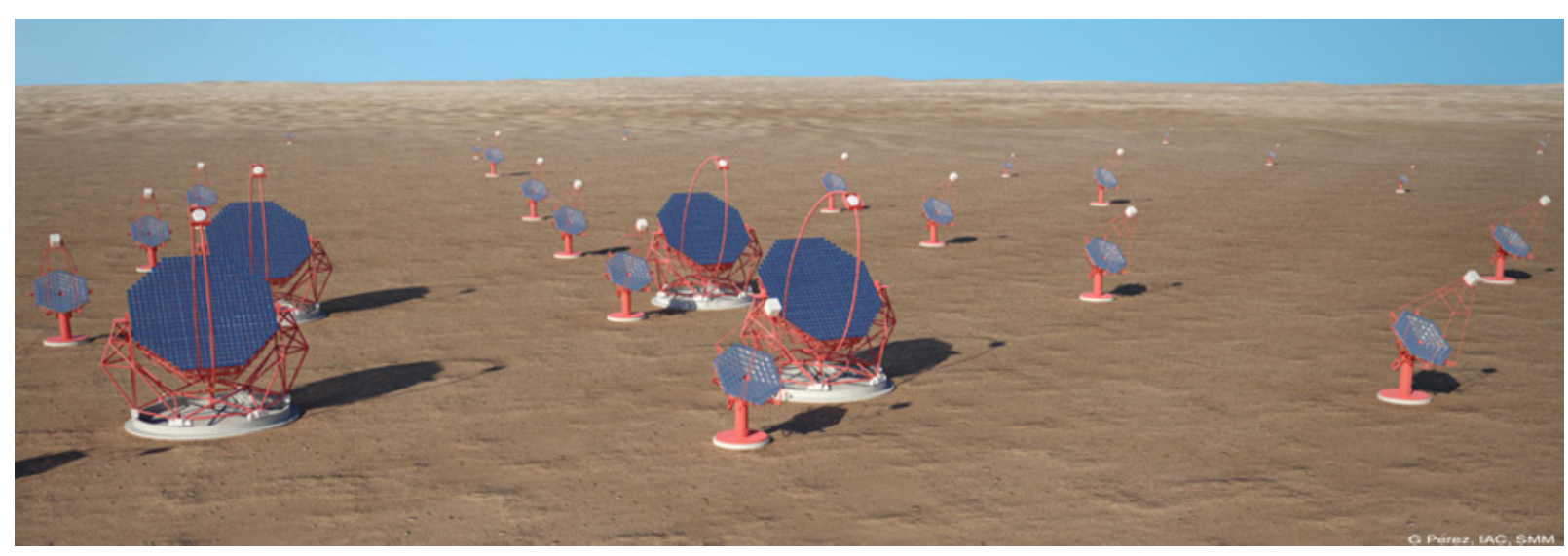

Figure 2. Artists impression of CTA (from http://www.cta-observatory.org/) .

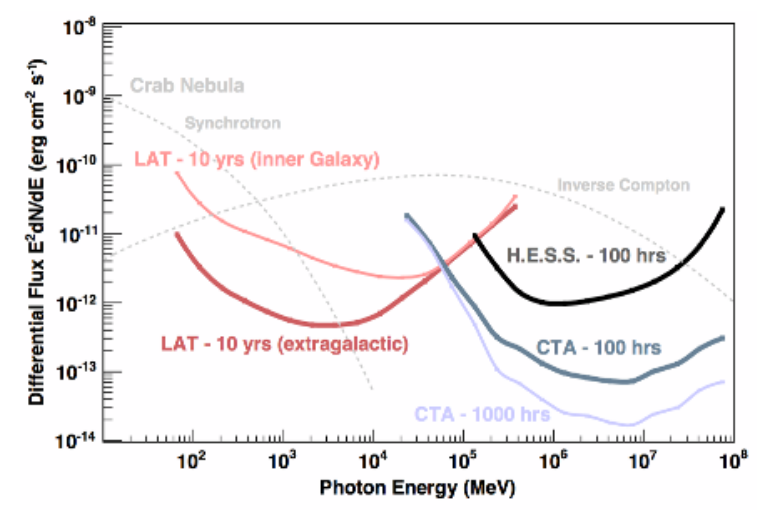

Figure 3. Expected point-source sensitivity of CTA in comparison with H.E.S.S. and the Fermi LAT. Four bins per decade of equal size on the logarithmic energy scale have been chosen for the calculation of the differential sensitivity. Figure taken from [15].

achieve the same sensitivity as with the "classical" geometrical reconstruction methods (e.g. [12-14]).

Non-imaging detectors measuring the shower particles reaching the ground measure continuously the sky at energies much greater than IACTs. Two such detector systems are currently in the construction phase: the HAWC system in Mexico ${ }^{4}$ and several instruments in Tibet (LAWCA, Tibet MD and LHASOO$^{5}$ ).

The next-generation gamma-ray observatory will be the Cherenkov Telescope Array ${ }^{6}$ (CTA; see Figure 2). It will consist of more than fifty IACTs of different size, covering a wide energy range from $20 \mathrm{GeV}$ to $200 \mathrm{TeV}$ with a factor of 10 improvement in sensitivity and a significantly improved angular resolution in comparison with current generation instruments. The different telescope types are optimized for different energy ranges. The lowest energies (10-100 GeV) will be covered by a few (3-4) large size telescopes with mirror areas $>400 \mathrm{~m}^{2}$. The limiting

\footnotetext{
${ }^{4}$ http://hawc.umd.edu/

${ }^{5}$ http://english.ihep.cas.cn/ic/ip/LHAASO/

${ }^{6}$ http://www.cta-observatory.org/
}

factors at these energies are photon collection efficiency, systematics and poor gamma-hadron separation. At energies above $100 \mathrm{GeV}$, mid-size telescopes with $100 \mathrm{~m}^{2}$ photon collection area will provide milli-Crab sensitivity and arcminute angular resolution. For high energies above 5-10 TeV, small telescopes distributed over a large area will be used to reach a collection area of more than $5 \times 10^{6}$ $\mathrm{m}^{2}$. CTA is currently in the prototyping phase. Its construction will start in 2015, first science data is expected for 2016. The expected sensitivity of CTA for steady point sources in comparison with current generation instruments and the Fermi LAT at lower energies can be seen from Figure 3. Additionally, CTA will open a new window for high-energy transients with flare durations of less than a week. As an example, at $75 \mathrm{GeV}$, CTA's sensitivity will result from its large sensitive area about a factor of $10^{4}$ $\left(10^{3}\right)$ greater than the current generation instruments for transient phenomena of 1 day (1 week) length [15].

\section{Supernova as sources of cosmic rays}

Cosmic rays are accelerated in the blast waves of Supernova remnants to energies of $10^{15} \mathrm{eV}$ and possibly beyond. The observation of gamma rays from SNRs give evidence of the environment in which acceleration take place, the efficiency and maximum energy of the accelerator. Three prominent examples of gamma-ray emitting SNRs are discussed in the following.

\subsection{Tycho}

Tycho Brahe observed in 1572 a supernova of type Ia in the constellation Cassiopeia. Tycho's supernova is located at a distance of 2-3 kpc with a current extension of about 8 '. High-resolution images by Chandra reveal strong nonthermal emission from the rim of the SNR and magnetic field strengths of up to $300 \mu \mathrm{G}$ implying magnetic field amplification from accelerated particles at these locations $[3,18]$. The shape and range of the non-thermal X-ray spectra indicate the presence of a multi-TeV electron population. Theoretical calculations show the cosmic ray acceleration should modify the structure of the of the rem- 

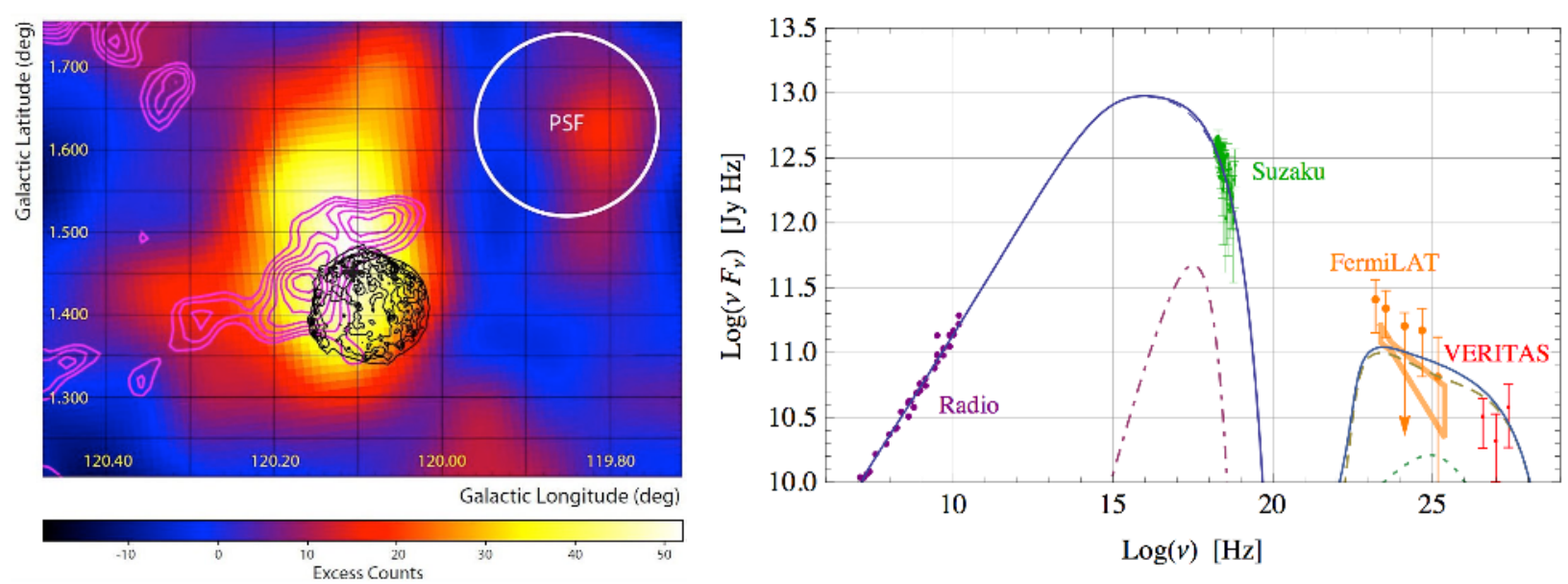

Figure 4. Left: TeV gamma-ray count map of the region around Tycho's SNR as measured by VERITAS [16]. The color scale indicate the number of excess events above $800 \mathrm{GeV}$. Overlaid are the X-ray image from a Chandra exposure and ${ }^{12} \mathrm{CO}$ emission from the FCRA survey (for references, see [16]). Right: broad-band spectral energy distribution of Tycho with measurements in radio, X-rays, $\mathrm{GeV}$ and $\mathrm{TeV}$ gamma rays (see [17] for references to the experimental data). Overlaid is a model curve from [17]. The curve shows synchrotron emission, thermal electron bremsstrahlung and pion decay.

nant. (e.g. [19]). Measurements of the location of the blast wave, the forward discontinuity and the reverse shock with Chandra confirm this and show that the shock dynamics are partly dominated by efficient cosmic acceleration [3].

Tycho has been recently discovered at gamma-ray energies by VERITAS [16] and the Fermi LAT [20], see Figure 4. Modeling of the broad-band spectral energy distribution confirms that hadrons are accelerated up to knee energies. The gamma-ray emission is likely dominated by the decay of neutral pions produced in collisions of accelerated cosmic rays with the background medium. The models predict that about $10 \%$ of the kinetic energy in the blast wave are converted into cosmic rays $[16,17,20]$. While hadronic models are favored for the description of the spectral energy distribution of Tycho, a leptonic origin of the high-energy emission cannot be excluded. Multizone models with regions of higher and lower magnetic field strengths can also provide a good fit to the gammaray fluxes of Tycho [21].

\subsection{RXJ 1713.7-3946}

RXJ 1713.7-3946 is a young ( $\approx 1600$ years old) shell-type SNR located at a distance of $1 \mathrm{kpc}$. It has a hard spectrum and is a bright gamma ray source with a flux above 1 $\mathrm{TeV}$ of approximately $65 \%$ of the flux of the Crab Nebula. HESS and Fermi observations show an extended shell-like emission region with spatially correlated gamma and Xray radiation $[22,23]$. Are these gamma rays signatures of hadronic cosmic rays? The situation is far from clear. Observations and modeling indicate that the gamma-ray emission in RXJ 1713.7-3946 is predominately leptonic, see for example the results from [24] in Figure 5. The non-observation of thermal X-ray emission from the SNR with Suzaku indicate very low gas densities of the circumstellar medium. The efficiency for gamma rays from decaying pions produced in proton-gas collisions is very low under the assumption of a uniform circumstellar medium and the high-energy photon originate from inverse Compton scattering of relativistic electrons on ambient photons. It should be stressed that in this leptonic model, about $25 \%$ of the shock ram kinetic energy flux goes into the acceleration of protons, while less than $1 \%$ into the highenergy electrons. A different and more detailed study of the molecular and atomic gas around RXJ 1713.7-3946 reports the observations of a low-density cavity created by the stellar wind of the progenitor with a surrounding denser wall [25]. The gamma rays are produced by protongas collisions in the dense wall while the low gas densities in the inner part explain the non-observation of thermal Xray emission by Suzaku. It is estimated from multi-zone hadronic modeling of RXJ 1713.7-3946 that about 0.1\% of the kinetic energy of the SNR is converted into highenergy particles [25]. The future ground-based observatory CTA will have an angular resolution of 1-2 arcminutes. It will have the potential to differentiate the location of the gamma-emission and to reveal the origin of highenergy emission from RXJ 1713.7-3946.

\subsection{Supernova remnants interacting with molecular clouds}

Dense clouds of molecular and atomic gas increase the observed gamma-ray flux from the generally radiative inefficient cosmic rays. The high densities support the production of neutral pions from proton-gas collisions. Two scenarios are possible: the direct interaction of the SNR's blast wave with the molecular cloud (e.g. [26]) and the interaction of the free-streaming cosmic rays with a nearby cloud (e.g. [27]). The first case involves an additional complication namely from the collision of the blast wave and its compression. Examples of interacting SNRs are W44, W51C, IC 443 and W28. All of them are detected at $\mathrm{GeV}$ and $\mathrm{TeV}$ energies. 


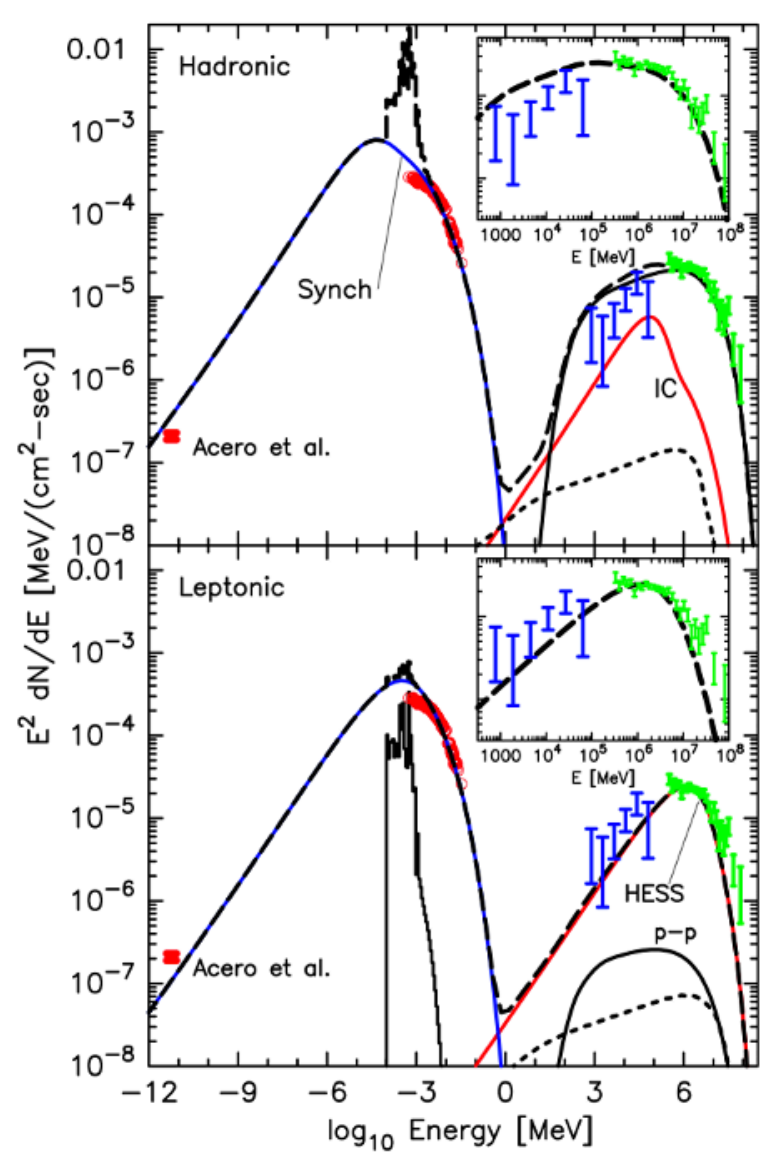

Figure 5. Broadband energy spectrum of RXJ 1713-396 with measurements by HESS and the Fermi LAT in gamma rays, Suzaku in $\mathrm{X}$-rays and at radio wavelengths (see [24] for references to the experimental data). A hadronic model is shown in the top panel and a leptonic model in the bottom panel [24].

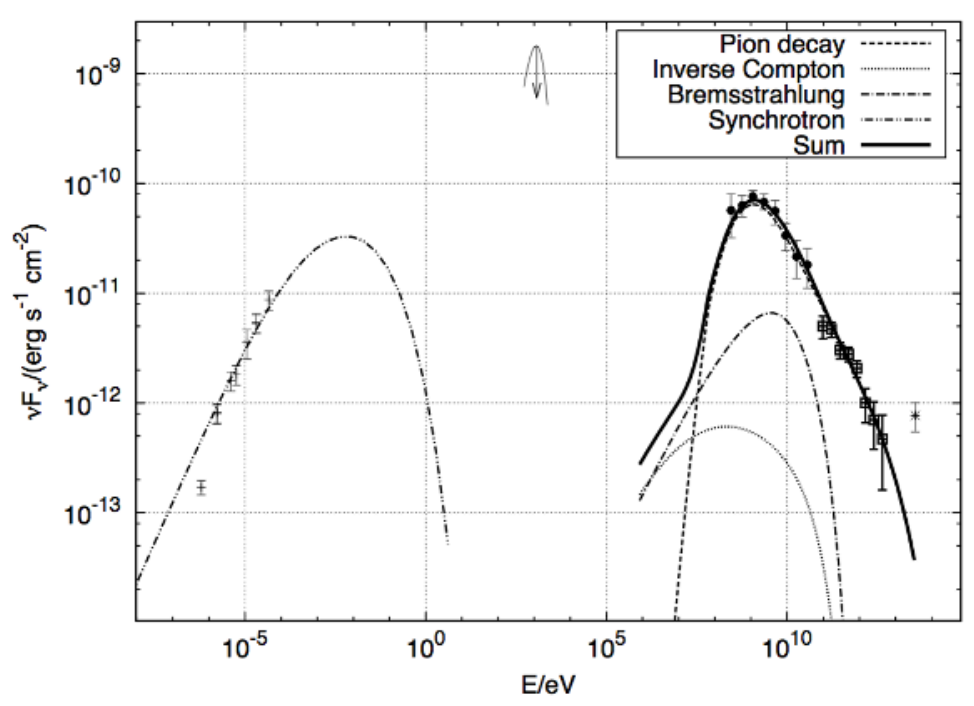

Figure 6. Broad-band spectral energy distribution of W51C with measurements in radio, GeV and TeV gamma rays (see [29] for references to the experimental data). Overlaid is a model curve of a hadronic dominated model from [29]. Data from MILAGRO (star) is ignored in the model fit. 
W51C is a radio bright SNR at a distance of $6 \mathrm{kpc}$ where the interaction of the shock with a cloud is observed [28]. Recent imaging of W51C by MAGIC and the Fermi LAT show a restricted emission zone where the SNR clearly interacts with the molecular cloud [29, 30]. The emission is best described by a hadronic model, see Figure 6 for the observational and modeling results. Contributions from bremsstrahlung, inverse Compton and pion decay are taken into account, protons with energies $>100$ $\mathrm{TeV}$ are needed to describe adequately the gamma-ray spectrum.

\section{Conclusion and outlook}

The combination of observations at all wavelengths and theoretical work established SNRs as main sources of cosmic rays. Many questions remain, not only regarding the details of cosmic ray acceleration in SNR as described above, but also the search for the sources of ultra-high energy cosmic rays and understanding of high-energy emitters like pulsars, blazars or binary systems. The current experimental situation in the field is unique: the Fermi LAT is taking data in its 5th year of operation and all groundbased instruments are entering a new phase with major upgrades just completed. The future is bright: new observatories like CTA and HAWC will answer and create many new questions of the high-energy Universe.

\section{Acknowledgements}

GM would like to thank the organizers of the ISVHECRI 2012 for the invitation to attend this interesting and inspiring conference in the heart of Berlin. GM acknowledges support through the Young Investigators Program of the Helmholtz Association.

\section{References}

[1] Hughes, J.P et al, ApJ 543 L61 (2000)

[2] Berezhko, E.G. \& Völk, H., A\&A 419 L27 (2004)

[3] Warren, J.S. et al, ApJ 634376 (2005)

[4] Acciari, V. et al (The VERITAS Collaboration), ApJ 714163 (2010)
[5] Weekes, T.C., arxiv:0811.1197 (2008)

[6] Hinton, J.A. \& Hofmann, W., ARA\&A 47523 (2009)

[7] Aharonian, F., Buckley, J, Kifune, T. \& Sinnis, G., Reports on Progress in Physics 71096901 (2008)

[8] Holder, J., arxiv:1204.1267 (2012)

[9] Ackermann, M. et al (The Fermi LAT collaboration), ApJS 2034 (2012)

[10] Weekes, T.C. et al, ApJ 342, 379 (1989)

[11] Punch, M. et al, Nature 358, 477 (1992)

[12] LeBohec, S. et al, NIM A 416, 425 (1998)

[13] de Naurois, M. \& Rolland, L., Astroparticle Journal 32, 231 (2009)

[14] Albert, J. et al, NIM A 588, 424 (2008)

[15] Funk, S. \& Hinton, J. (CTA Collaboration), Astroparticle Journal, accepted (2012)

[16] Acciari, V. et al (The VERITAS Collaboration), ApJ 730 L20 (2011)

[17] Morlino, G. \& Caprioli, D., A\&A 538 A81 (2012)

[18] Hwang, U. et al, ApJ 581, 1101 (2002)

[19] Bell, A.R. \& Lucek, S.G. MNRAS 321, 433 (2001)

[20] Giordano, F. et al. (for the Fermi-LAT collaboration) PoS, proceedings of the 25th Texas Symposium, Heidelberg, Germany (2010)

[21] Atyoan, A. and Dermer, C.D., ApJ 749, L26 (2012)

[22] Aharonian, F. et al(The H.E.S.S. collaboration) A\&A 449, 223 (2006)

[23] Abdo, A.A. et al( The Fermi LAT collaboration) ApJ 734, 28 (2011)

[24] Ellison, D.C. et al, ApJ 712, 287 (2010)

[25] Fukui, Y. et al, ApJ 746, 82 (2012)

[26] Uchiyama, Y. et al, ApJ 723, L22 (2010)

[27] Aharonian, F. A., \& Atoyan, A. M., A\&A, 309, 917 (1996)

[28] Koo, B.-C., \& Moon, D.-S., ApJ, 475, 194 (1997)

[29] Aleksic, J. et al (The MAGIC Collaboration), A\&A 541, A13 (2012)

[30] Abdo, A.A. et al (The Fermi LAT Collaboration), ApJ 706, L1 (2012) 